\title{
QUOTIENT SPACES AND THE OPEN MAP THEOREM
}

\author{
BY W. S£OWIKOWSKI ${ }^{1}$
}

Communicated by Einar Hille, May 17, 1961

It has been shown by Köthe [1] and Grothendieck [2] that the operation of taking the quotient space does not preserve several important properties of the initial space if we leave the class of metrizable spaces. In this paper the technique of quotient spaces is used in sketching a proof of a theorem similar to that about the open map but expressed in terms of the concepts introduced in [3]. It is assumed that the reader is familiar with [3].

The theorem can be directly used to secure some necessary and sufficient conditions for continuity of functionals bounded on subspaces of (LF)-spaces but that will be shown in a separate paper.

Let us consider an $\alpha \beta \gamma$-representation $\mathfrak{F}=\left(N, X_{k, n},|\cdot|_{k, n}\right)$. We can always arrange for $k$ and $n$ to run over the set of natural numbers.

$\mathfrak{F}$ is said to be complete if all spaces $X_{\left(k_{n}\right)},|\cdot|_{\left(k_{n}\right)},\left(k_{n}\right) \in N$ are complete (see [3]); $\mathfrak{F}$ is said to be directed if for arbitrary $\left(k_{n}^{(i)}\right) \in N$, $i=1,2$ there is $\left(k_{n}^{(3)}\right) \in N$ such that $X_{\left(k_{n}{ }^{(i)}\right)} \subset X_{\left(k_{n}{ }^{(3)}\right)}$ for $i=1,2$ and the identical imbeddings are continuous with respect to the proper $|\cdot|{ }_{\left({ }_{k_{n}}\left({ }^{(i)}\right)\right.}, i=1,2,3$. Denote $S=\left\{\left(k_{1}, \cdots, k_{p}\right)\right.$ : there are $k_{p+1}, k_{p+2}, \cdots$ such that $\left.\left(k_{n}\right) \in N\right\}$ and for $s=\left(k_{1}, \cdots, k_{p}\right) \in S$ let $X_{s, p}=X_{k_{1}, 1} \cap \cdots$ $\cap X_{k_{p}, p}$ and

$$
|x|_{s, p}=\max _{1 \leqq i \leqq p}|x|_{k_{i}, i} .
$$

Let in the following $\hat{N}=\left\{\left(s_{n}\right)\right.$ : there is $\left(k_{n}\right) \in N$ such that $s_{p}$ $\left.=\left(k_{1}, \cdots, k_{p}\right), p=1,2, \cdots\right\}$. It is easy to see that $\hat{\mathfrak{F}}=\left(\hat{N}, X_{s, n},|\cdot|_{s, n}\right)$ is an $\alpha \beta \gamma$-representation. $\hat{\mathfrak{F}}$ is said to be an alternative form of $\mathfrak{F}$. Notice that we have now

$$
X_{s_{p+1}, p+1} \subset X_{s_{p}, p} \text { and }|x|_{s_{p}, p} \leqq|x|_{s_{p+1}, p+1}
$$

for $\left(s_{n}\right) \in \hat{N}$ and $x \in X_{s_{p+1}, p+1}, p=1,2, \cdots$. Moreover, $X_{\left(s_{n}\right)}=X_{\left(k_{n}\right)}$ and $|\cdot|_{\left(k_{n}\right)}$ is equivalent to $|\cdot|_{\left(s_{n}\right)}$ for $s_{p}=\left(k_{1}, \cdots, k_{p}\right)$ and $\left(k_{n}\right) \in N$.

Let $K$ be a linear subset of $X=\bigcup_{N} X_{\left(k_{n}\right)}=\bigcup_{\hat{N}} X_{\left(s_{n}\right)}$. Denote $X_{s, n}^{K}=X_{s, n} / K \subset X / K$ and $|x / K|_{s, n}^{K}=\inf \left\{|x+y|_{s, n}: y \in X_{s, n} \cap K\right\}$, where $x \in X_{s, n}$ and $s \in S$. Let further $X_{\left(s_{n}\right)}^{K}=\bigcap_{n=1}^{\infty} X_{s_{n}, n}^{K}$ for $\left(s_{n}\right) \in \hat{N}$ and $|x|_{\left(s_{n}\right)}^{K}=\sum_{n=1}^{\infty} 2^{-n}|x|_{s_{n}, n}^{K}\left(1+|x|_{s_{n}, n}^{K}\right)^{-1}$. Suppose $Z$, \|. $\|$ is a complete normed space and $Z$ is a linear subset of $X / K$ (the homogeneity of $\|\cdot\|$ is not assumed).

\footnotetext{
${ }^{1}$ Supported by National Science Foundation Grant G-14600.
} 
Theorem. Suppose that $\mathfrak{F}$ is complete and directed. If for each sequence $\left(x_{n} / K\right) \subset X_{\left(s_{n}\right)}^{K} \cap Z$ the condition

$$
\lim _{n}\left\|x_{n} / K-x_{1} / K\right\|=\lim _{m}\left|x_{m} / K-x_{2} / K\right|_{\left(s_{n}\right)}^{K}=0
$$

implies $x_{1}-x_{2} \in K$, then there is $\left(s_{n}^{0}\right) \in \hat{N}$ such that $Z \subset X_{\left(\mathbf{s}_{\mathbf{n}}^{0}\right)}^{K}$ and $\lim _{n}\left\|x_{n} / K\right\|=0$ implies $\lim _{n}\left|x_{n} / K\right|_{\left(s_{n}\right)}^{K}=0$.

To prove the theorem we will need the following:

Lemma. If for given $\left(s_{n}\right) \in N$ and $\left(x_{n}\right) \subset X$ we have $\sum_{n=1}^{\infty}\left|x_{n+1} / K-x_{n} / K\right|_{s_{n}, n}^{K}<\infty$, then there is $x_{0} \in X$ such that $x_{0} / K$ $-x_{n} / K \in X_{\boldsymbol{s}_{q}, q} / K$ for $n \geqq q$ and $\lim _{n}\left|x_{0} / K-x_{n} / K\right|_{s_{q}, q}^{K}=0$ for $q=1,2, \cdots$.

ProOF. We have $\sum_{n=1}^{\infty}\left|x_{n+1}-x_{n}+y_{n}\right|_{s_{n}, n}<\infty$ for some $y_{n} \in K$ such that $x_{n+1}-x_{n}+q_{n} \in X_{s_{n}, n}$ for $n=1,2, \cdots$. By $\gamma$ (see [3]) there are $\left(s_{n}^{q}\right) \in \hat{N}, q=1,2, \cdots \quad$ such that $s_{q}=s_{q}^{q}$ for $q=1,2, \cdots, x_{p+1}-x_{p}$ $+y_{n} \in X_{\left(s_{n}\right)}$, for $p \geqq q$ and

$$
\lim _{n>q}\left(\sup _{k}\left|\sum_{i=0}^{k}\left(x_{n+i+1}-x_{n+i}+y_{n+i}\right)\right|_{\left(s_{n}{ }^{q}\right)}\right)=0 .
$$

This means that

$$
\lim _{n}\left(\sup _{k}\left|x_{n+k+1}-x_{n}+\sum_{j=n}^{n+k} y_{j}\right|_{\left(\boldsymbol{s}_{n}{ }^{q}\right)}\right)=0
$$

or

$$
\left.\lim _{n} \sup _{k}\left|\left(x_{n+k+1}+\sum_{j=q}^{n+k} y_{j}\right)-\left(x_{n}+\sum_{j=q}^{n-1} y_{j}\right)\right|_{\left(s_{n}^{q}\right)}\right)=0
$$

and

$$
u_{p}-\sum_{j=1}^{4} y_{j}-x_{q} \in X_{\left(s_{n}\right)}^{q} \quad \text { for } p>q
$$

where

$$
u_{p}=x_{p}+\sum_{j=1}^{p-1} y_{j}, \quad \quad p=2,3, \cdots
$$

Thus, for fixed $q, u_{p}-\sum_{j=1}^{o} y_{j}-x_{q}$ tends to some limit in $X_{\left(s_{n}{ }^{q}\right)}$. But $\mathfrak{F}$ is directed and then $\left(u_{p}\right)$ tends to $x_{0}$ in some $X_{\left(8_{n}^{0}\right)}^{0},|\cdot|{ }_{\left(s_{n}^{0}\right)}^{0}$ and $u_{p}-\sum_{j=1}^{q} y_{j}-x_{q}$ tends to 


$$
x_{0}-\sum_{j=1}^{q} y_{j}-x_{q} \in X_{\left(s_{n}{ }^{q}\right)} \subset X_{s_{q}, q} .
$$

Therefore $x_{0} / K-x_{n} / K \in X_{s_{q}, q} / K$ for $n \geqq q$. Moreover

$$
\begin{aligned}
\lim _{n}\left|x_{n} / K-x_{0} / K\right|_{8_{q}, q}^{K} & \\
& \leqq \lim _{n}\left|\left(x_{n}+\sum_{j=q}^{n-1} y_{j}\right)-\left(x_{0}-\sum_{j=1}^{q-1} y_{j}\right)\right|_{\delta_{q}, q}=0
\end{aligned}
$$

and the lemma holds.

Proof of the Theorem. Consider the $\alpha \beta \gamma$-representation $\hat{\mathfrak{F}} / K$ $=\left(\hat{N}, X_{s, n}^{K},|\cdot|{ }_{s, n}^{K}\right)$. From the lemma follows that $\hat{\mathfrak{F}} / K$ is complete. Hence $Z,\|\cdot\|$ and $\hat{F} / K$ are subject to the theorem proved in [3].

Applying this theorem we obtain the desired result.

\section{REFERENCES}

1. G. Köthe, Die Quotientenräume eines linearen vollkommenen Raumes, Math. Z. vol. 51 (1947) pp. 17-35.

2. A. Grothendieck, Sur les espaces $(F)$ et $(D F)$, Summa Brasil. Math. vol. 3 (1954) pp. 57-123.

3. W. Słowikowski, On continuity of inverse operators, Bull. Amer. Math. Soc. vol. 67 (1961) pp. 467-470.

The Institute for Advanced Study 